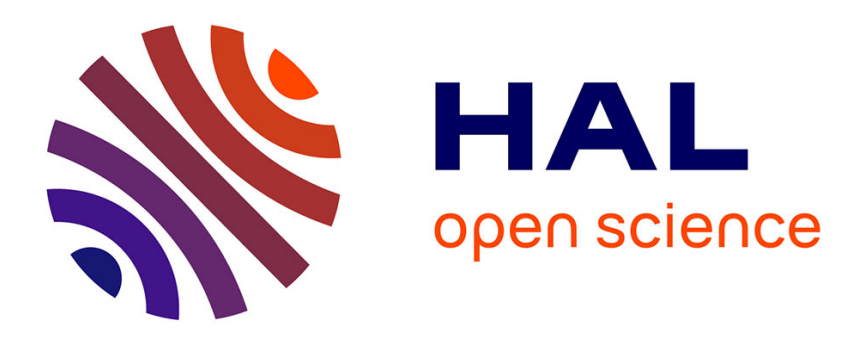

\title{
Highly Undecidable Problems about Recognizability by Tiling Systems
}

\author{
Olivier Finkel
}

\section{To cite this version:}

Olivier Finkel. Highly Undecidable Problems about Recognizability by Tiling Systems. Fundamenta Informaticae, 2009, 91 (2), pp.305-323. ensl-00340791

\section{HAL Id: ensl-00340791}

\section{https://hal-ens-lyon.archives-ouvertes.fr/ensl-00340791}

Submitted on 22 Nov 2008

HAL is a multi-disciplinary open access archive for the deposit and dissemination of scientific research documents, whether they are published or not. The documents may come from teaching and research institutions in France or abroad, or from public or private research centers.
L'archive ouverte pluridisciplinaire HAL, est destinée au dépôt et à la diffusion de documents scientifiques de niveau recherche, publiés ou non, émanant des établissements d'enseignement et de recherche français ou étrangers, des laboratoires publics ou privés. 


\title{
Highly Undecidable Problems about Recognizability by Tiling Systems
}

\author{
Olivier Finkel \\ Equipe Modèles de Calcul et Complexité \\ Laboratoire de l'Informatique du Parallélisme \\ CNRS et Ecole Normale Supérieure de Lyon \\ 46, Allée d'Italie 69364 Lyon Cedex 07, France. \\ Olivier.Finkel@ens-lyon.fr
}

\begin{abstract}
Altenbernd, Thomas and Wöhrle have considered acceptance of languages of infinite two-dimensional words (infinite pictures) by finite tiling systems, with usual acceptance conditions, such as the Büchi and Muller ones, in [1]. It was proved in [9] that it is undecidable whether a Büchirecognizable language of infinite pictures is E-recognizable (respectively, A-recognizable). We show here that these two decision problems are actually $\Pi_{2}^{1}$-complete, hence located at the second level of the analytical hierarchy, and "highly undecidable". We give the exact degree of numerous other undecidable problems for Büchi-recognizable languages of infinite pictures. In particular, the nonemptiness and the infiniteness problems are $\Sigma_{1}^{1}$-complete, and the universality problem, the inclusion problem, the equivalence problem, the determinizability problem, the complementability problem, are all $\Pi_{2}^{1}$-complete. It is also $\Pi_{2}^{1}$-complete to determine whether a given Büchi recognizable language of infinite pictures can be accepted row by row using an automaton model over ordinal words of length $\omega^{2}$.
\end{abstract}

Keywords: Languages of infinite pictures; recognizability by tiling systems; decision problems; highly undecidable problems; analytical hierarchy.

\section{Introduction}

Languages of infinite words accepted by finite automata were first studied by Büchi to prove the decidability of the monadic second order theory of one successor over the integers. Since then regular $\omega$-languages have been much studied and many applications have been found for specification and verification of non-terminating systems, see [24, 23, 19] for many results and references.

Address for correspondence: E Mail: Olivier.Finkel@ens-lyon.fr 
In a recent paper, Altenbernd, Thomas and Wöhrle have considered acceptance of languages of infinite two-dimensional words (infinite pictures) by finite tiling systems, with the usual acceptance conditions, such as the Büchi and Muller ones, firstly used for infinite words. This way they extended both the classical theory of $\omega$-regular languages and the classical theory of recognizable languages of finite pictures, [11], to the case of infinite pictures.

Many classical decision problems are studied in formal language theory and in automata theory and arise now naturally about recognizable languages of infinite pictures. We proved in [9] that many decision problems for Büchi-recognizable languages of infinite pictures are undecidable. In particular, we showed, using topological arguments, that it is undecidable whether a Büchi-recognizable language of infinite pictures is E-recognizable (respectively, A-recognizable), giving the answer to two questions raised in [1]. We proved also several other undecidability results as the following ones: one cannot decide whether a Büchi-recognizable language of infinite pictures can be recognized by a deterministic Büchi or Muller tiling system, or whether it can be accepted row by row using an automaton model over ordinal words of length $\omega^{2}$.

Using the $\Pi_{2}^{1}$-completeness of the universality problem for $\omega$-languages of non deterministic Turing machines which was proved by Castro and Cucker in [B], and some topological arguments, we show in this paper that the above decision problems are actually $\Pi_{2}^{1}$-complete, hence located at the second level of the analytical hierarchy, and "highly undecidable". Using other results of [3], we give also the exact degree of numerous other undecidable problems for Büchi-recognizable languages of infinite pictures. In particular, the non-emptiness and the infiniteness problems are $\Sigma_{1}^{1}$-complete, and the universality problem, the inclusion problem, the equivalence problem, the complementability problem, are all $\Pi_{2}^{1}-$ complete. This gives new natural examples of decision problems located at the first or at the second level of the analytical hierarchy. We show also that topological properties of Büchi-recognizable languages of infinite pictures are highly undecidable.

The paper is organized as follows. In Section 2 we recall definitions for pictures and tiling systems. The definition and properties of the analytical hierarchy are introduced in Section 3. We recall in Section 4 some notions of topology, including the definitions of Borel and analytic sets. We prove high undecidability results in Section 5. Concluding remarks are given in Section 6.

\section{Tiling Systems}

We assume the reader to be familiar with the theory of formal $(\omega)$-languages [24, 23]. We recall usual notations of formal language theory.

When $\Sigma$ is a finite alphabet, a non-empty finite word over $\Sigma$ is any sequence $x=a_{1} \ldots a_{k}$, where $a_{i} \in \Sigma$ for $i=1, \ldots, k$, and $k$ is an integer $\geq 1$. The length of $x$ is $k$, denoted by $|x|$. The empty word has no letter and is denoted by $\lambda$; its length is $0 . \Sigma^{\star}$ is the set of finite words (including the empty word) over $\Sigma$. The first infinite ordinal is $\omega$. An $\omega$-word over $\Sigma$ is an $\omega$-sequence $a_{1} \ldots a_{n} \ldots$, where for all integers $i \geq 1, \quad a_{i} \in \Sigma$. When $\sigma$ is an $\omega$-word over $\Sigma$, we write $\sigma=\sigma(1) \sigma(2) \ldots \sigma(n) \ldots$, where for all $i, \sigma(i) \in \Sigma$, and $\sigma[n]=\sigma(1) \sigma(2) \ldots \sigma(n)$ for all $n \geq 1$ and $\sigma[0]=\lambda$.

The usual concatenation product of two finite words $u$ and $v$ is denoted $u . v$ (and sometimes just $u v$ ). 
This product is extended to the product of a finite word $u$ and an $\omega$-word $v$ : the infinite word $u . v$ is then the $\omega$-word such that:

$(u . v)(k)=u(k)$ if $k \leq|u|$, and $(u \cdot v)(k)=v(k-|u|)$ if $k>|u|$.

The set of $\omega$-words over the alphabet $\Sigma$ is denoted by $\Sigma^{\omega}$. An $\omega$-language over an alphabet $\Sigma$ is a subset of $\Sigma^{\omega}$.

We now define two-dimensional words, i.e. pictures.

Let $\Sigma$ be a finite alphabet and \# be a letter not in $\Sigma$ and let $\hat{\Sigma}=\Sigma \cup\{\#\}$. If $m$ and $n$ are two integers $>0$ or if $m=n=0$, a picture of size $(m, n)$ over $\Sigma$ is a function $p$ from $\{0,1, \ldots, m+1\} \times\{0,1, \ldots, n+1\}$ into $\hat{\Sigma}$ such that $p(0, i)=p(m+1, i)=\#$ for all integers $i \in\{0,1, \ldots, n+1\}$ and $p(i, 0)=$ $p(i, n+1)=\#$ for all integers $i \in\{0,1, \ldots, m+1\}$ and $p(i, j) \in \Sigma$ if $i \notin\{0, m+1\}$ and $j \notin\{0, n+1\}$. The empty picture is the only picture of size $(0,0)$ and is denoted by $\lambda$. Pictures of size $(n, 0)$ or $(0, n)$, for $n>0$, are not defined. $\Sigma^{\star, \star}$ is the set of pictures over $\Sigma$. A picture language $L$ is a subset of $\Sigma^{\star, \star}$.

An $\omega$-picture over $\Sigma$ is a function $p$ from $\omega \times \omega$ into $\hat{\Sigma}$ such that $p(i, 0)=p(0, i)=\#$ for all $i \geq 0$ and $p(i, j) \in \Sigma$ for $i, j>0$. $\Sigma^{\omega, \omega}$ is the set of $\omega$-pictures over $\Sigma$. An $\omega$-picture language $L$ is a subset of $\Sigma^{\omega, \omega}$.

For $\Sigma$ a finite alphabet we call $\Sigma^{\omega^{2}}$ the set of functions from $\omega \times \omega$ into $\Sigma$. So the set $\Sigma^{\omega, \omega}$ of $\omega$-pictures over $\Sigma$ is a strict subset of $\hat{\Sigma}^{\omega^{2}}$.

We shall say that, for each integer $j \geq 1$, the $j^{\text {th }}$ row of an $\omega$-picture $p \in \Sigma^{\omega, \omega}$ is the infinite word $p(1, j) \cdot p(2, j) \cdot p(3, j) \ldots$ over $\Sigma$ and the $j^{\text {th }}$ column of $p$ is the infinite word $p(j, 1) \cdot p(j, 2) \cdot p(j, 3) \ldots$ over $\Sigma$.

As usual, one can imagine that, for integers $j>k \geq 1$, the $j^{\text {th }}$ column of $p$ is on the right of the $k^{\text {th }}$ column of $p$ and that the $j^{\text {th }}$ row of $p$ is "above" the $k^{\text {th }}$ row of $p$.

We introduce now tiling systems as in the paper [1]].

A tiling system is a tuple $\mathcal{A}=(Q, \Sigma, \Delta)$, where $Q$ is a finite set of states, $\Sigma$ is a finite alphabet, $\Delta \subseteq$ $(\hat{\Sigma} \times Q)^{4}$ is a finite set of tiles.

A Büchi tiling system is a pair $(\mathcal{A}, F)$ where $\mathcal{A}=(Q, \Sigma, \Delta)$ is a tiling system and $F \subseteq Q$ is the set of accepting states.

A Muller tiling system is a pair $(\mathcal{A}, \mathcal{F})$ where $\mathcal{A}=(Q, \Sigma, \Delta)$ is a tiling system and $\mathcal{F} \subseteq 2^{Q}$ is the set of accepting sets of states.

Tiles are denoted by $\left(\begin{array}{ll}\left(a_{3}, q_{3}\right) & \left(a_{4}, q_{4}\right) \\ \left(a_{1}, q_{1}\right) & \left(a_{2}, q_{2}\right)\end{array}\right)$ with $a_{i} \in \hat{\Sigma}$ and $q_{i} \in Q$,

and in general, over an alphabet $\Gamma$, by $\left(\begin{array}{cc}b_{3} & b_{4} \\ b_{1} & b_{2}\end{array}\right) \quad$ with $b_{i} \in \Gamma$.

A combination of tiles is defined by:

$$
\left(\begin{array}{ll}
b_{3} & b_{4} \\
b_{1} & b_{2}
\end{array}\right) \circ\left(\begin{array}{ll}
b_{3}^{\prime} & b_{4}^{\prime} \\
b_{1}^{\prime} & b_{2}^{\prime}
\end{array}\right)=\left(\begin{array}{ll}
\left(b_{3}, b_{3}^{\prime}\right) & \left(b_{4}, b_{4}^{\prime}\right) \\
\left(b_{1}, b_{1}^{\prime}\right) & \left(b_{2}, b_{2}^{\prime}\right)
\end{array}\right)
$$


A run of a tiling system $\mathcal{A}=(Q, \Sigma, \Delta)$ over a (finite) picture $p$ of size $(m, n)$ over $\Sigma$ is a mapping $\rho$ from $\{0,1, \ldots, m+1\} \times\{0,1, \ldots, n+1\}$ into $Q$ such that for all $(i, j) \in\{0,1, \ldots, m\} \times\{0,1, \ldots, n\}$ with $p(i, j)=a_{i, j}$ and $\rho(i, j)=q_{i, j}$ we have

$$
\left(\begin{array}{cc}
a_{i, j+1} & a_{i+1, j+1} \\
a_{i, j} & a_{i+1, j}
\end{array}\right) \circ\left(\begin{array}{cc}
q_{i, j+1} & q_{i+1, j+1} \\
q_{i, j} & q_{i+1, j}
\end{array}\right) \in \Delta .
$$

A run of a tiling system $\mathcal{A}=(Q, \Sigma, \Delta)$ over an $\omega$-picture $p \in \Sigma^{\omega, \omega}$ is a mapping $\rho$ from $\omega \times \omega$ into $Q$ such that for all $(i, j) \in \omega \times \omega$ with $p(i, j)=a_{i, j}$ and $\rho(i, j)=q_{i, j}$ we have

$$
\left(\begin{array}{cc}
a_{i, j+1} & a_{i+1, j+1} \\
a_{i, j} & a_{i+1, j}
\end{array}\right) \circ\left(\begin{array}{cc}
q_{i, j+1} & q_{i+1, j+1} \\
q_{i, j} & q_{i+1, j}
\end{array}\right) \in \Delta .
$$

We now recall acceptance of finite or infinite pictures by tiling systems:

Definition 2.1. Let $\mathcal{A}=(Q, \Sigma, \Delta)$ be a tiling system, $F \subseteq Q$ and $\mathcal{F} \subseteq 2^{Q}$.

- The picture language recognized by $\mathcal{A}$ is the set of pictures $p \in \Sigma^{\star, \star}$ such that there is some run $\rho$ of $\mathcal{A}$ on $p$.

- The $\omega$-picture language A-recognized (respectively, E-recognized, Büchi-recognized) by $(\mathcal{A}, F)$ is the set of $\omega$-pictures $p \in \Sigma^{\omega, \omega}$ such that there is some run $\rho$ of $\mathcal{A}$ on $p$ and $\rho(v) \in F$ for all (respectively, for at least one, for infinitely many) $v \in \omega^{2}$. It is denoted by $L^{A}((\mathcal{A}, F))$ (respectively, $\left.L^{E}((\mathcal{A}, F)), L^{B}((\mathcal{A}, F))\right)$.

- The $\omega$-picture language Muller-recognized by $(\mathcal{A}, \mathcal{F})$ is the set of $\omega$-pictures $p \in \Sigma^{\omega, \omega}$ such that there is some run $\rho$ of $\mathcal{A}$ on $p$ and $\operatorname{In} f(\rho) \in \mathcal{F}$ where $\operatorname{Inf}(\rho)$ is the set of states occurring infinitely often in $\rho$. It is denoted by $L^{M}((\mathcal{A}, \mathcal{F}))$.

Notice that an $\omega$-picture language $L \subseteq \Sigma^{\omega, \omega}$ is recognized by a Büchi tiling system if and only if it is recognized by a Muller tiling system, [1].

We shall denote $T S\left(\Sigma^{\omega, \omega}\right)$ the class of languages $L \subseteq \Sigma^{\omega, \omega}$ which are recognized by some Büchi (or Muller) tiling system.

We recall now an interesting variation of the above defined acceptance conditions for infinite pictures, introduced in [1]. This variation uses the diagonal of an $\omega$-picture.

The diagonal of an $\omega$-picture $p$ is the set of vertices $D i(p)=\{(i, i) \mid i \in \omega\}$.

The $\omega$-picture language A-recognized (respectively, E-recognized, Büchi-recognized) by $(\mathcal{A}, F)$ on the diagonal is the set of $\omega$-pictures $p \in \Sigma^{\omega, \omega}$ such that there is some run $\rho$ of $\mathcal{A}$ on $p$ and $\rho(v) \in F$ for all (respectively, for at least one, for infinitely many) $v \in D i(p)$.

We define similarly the notion of $\omega$-picture language Muller-recognized on the diagonal by $(\mathcal{A}, \mathcal{F})$, replacing $\operatorname{In} f(\rho)$ by the set of states $\operatorname{In} f(D i(\rho))$ occurring infinitely often on the diagonal of $\rho$.

The following result was stated in [1]]. 
Theorem 2.2. An $\omega$-picture language $L \subseteq \Sigma^{\omega, \omega}$ is A-recognized (respectively, E-recognized, Büchirecognized, Muller-recognized) by a tiling system if and only if it is A-recognized (respectively, Erecognized, Büchi-recognized, Muller-recognized) on the diagonal by a tiling system.

We wish now to see links with classical notions of tiling of the (quarter of the) plane, see for instance [2].

We denote $\Gamma=\hat{\Sigma} \times Q$ where $\Sigma$ is the alphabet of pictures and $Q$ is the set of states of a tiling system $\mathcal{A}=(Q, \Sigma, \Delta)$. We consider configurations which are elements of $\Gamma^{\omega \times \omega}$. One can imagine that each cell of the quarter of the plane contains a letter of the alphabet $\Gamma$.

Let $\Delta \subseteq(\hat{\Sigma} \times Q)^{4}=\Gamma^{4}$ be a finite set of tiles. We denote its complement by $\Delta^{-}=\Gamma^{4}-\Delta$. A tiling of the (quarter of the) plane with $\Delta^{-}$as set of forbidden patterns is simply a configuration $c \in \Gamma^{\omega \times \omega}$ such that for all integers $i, j \in \omega$ :

$$
\left(\begin{array}{cc}
c(i, j+1) & c(i+1, j+1) \\
c(i, j) & c(i+1, j)
\end{array}\right) \in \Delta .
$$

Then the $\omega$-picture language $L \subseteq \Sigma^{\omega, \omega}$ which is A-recognized (respectively, E-recognized, Büchirecognized) on the diagonal by the tiling system $(\mathcal{A}, F)$ is simply the set of $\omega$-pictures $p \in \Sigma^{\omega, \omega}$ which are projections of configurations $c \in \Gamma^{\omega \times \omega}$ which are tilings of the (quarter of the) plane with $\Delta^{-}$as set of forbidden patterns such that for all (respectively, for at least one, for infinitely many) $i \in \omega$ the second component of $c(i, i)$ is in $F$. A similar characterization can be given for the Muller acceptance condition.

We can also easily state similar characterizations for global recognizability, i.e. not on the diagonal, by tiling systems.

\section{The Analytical Hierarchy}

The set of natural numbers is denoted by $\mathbb{N}$ and the set of all mappings from $\mathbb{N}$ into $\mathbb{N}$ will be denoted by $\mathcal{F}$.

We assume the reader to be familiar with the arithmetical hierarchy on subsets of $\mathbb{N}$. We now recall the notions of analytical hierarchy and of complete sets for classes of this hierarchy which may be found in [21].

Definition 3.1. Let $k, l>0$ be some integers. $\Phi$ is a partial recursive function of $k$ function variables and $l$ number variables if there exists $z \in \mathbb{N}$ such that for any $\left(f_{1}, \ldots, f_{k}, x_{1}, \ldots, x_{l}\right) \in \mathcal{F}^{k} \times \mathbb{N}^{l}$, we have

$$
\Phi\left(f_{1}, \ldots, f_{k}, x_{1}, \ldots, x_{l}\right)=\tau_{z}^{f_{1}, \ldots, f_{k}}\left(x_{1}, \ldots, x_{l}\right),
$$

where the right hand side is the output of the Turing machine with index $z$ and oracles $f_{1}, \ldots, f_{k}$ over the input $\left(x_{1}, \ldots, x_{l}\right)$. For $k>0$ and $l=0, \Phi$ is a partial recursive function if, for some $z$,

$$
\Phi\left(f_{1}, \ldots, f_{k}\right)=\tau_{z}^{f_{1}, \ldots, f_{k}}(0) .
$$

The value $z$ is called the Gödel number or index for $\Phi$. 
Definition 3.2. Let $k, l>0$ be some integers and $R \subseteq \mathcal{F}^{k} \times \mathbb{N}^{l}$. The relation $R$ is said to be a recursive relation of $k$ function variables and $l$ number variables if its characteristic function is recursive.

We now define analytical subsets of $\mathbb{N}^{l}$.

Definition 3.3. A subset $R$ of $\mathbb{N}^{l}$ is analytical if it is recursive or if there exists a recursive set $S \subseteq$ $\mathcal{F}^{m} \times \mathbb{N}^{n}$, with $m \geq 0$ and $n \geq l$, such that

$$
R=\left\{\left(x_{1}, \ldots, x_{l}\right) \mid\left(Q_{1} s_{1}\right)\left(Q_{2} s_{2}\right) \ldots\left(Q_{m+n-l} s_{m+n-l}\right) S\left(f_{1}, \ldots, f_{m}, x_{1}, \ldots, x_{n}\right)\right\},
$$

where $Q_{i}$ is either $\forall$ or $\exists$ for $1 \leq i \leq m+n-l$, and where $s_{1}, \ldots, s_{m+n-l}$ are $f_{1}, \ldots, f_{m}, x_{l+1}, \ldots, x_{n}$ in some order.

The expression $\left(Q_{1} s_{1}\right)\left(Q_{2} s_{2}\right) \ldots\left(Q_{m+n-l} s_{m+n-l}\right) S\left(f_{1}, \ldots, f_{m}, x_{1}, \ldots, x_{n}\right)$ is called a predicate form for $R$. A quantifier applying over a function variable is of type 1 , otherwise it is of type 0 . In a predicate form the (possibly empty) sequence of quantifiers, indexed by their type, is called the prefix of the form. The reduced prefix is the sequence of quantifiers obtained by suppressing the quantifiers of type 0 from the prefix.

The levels of the analytical hierarchy are distinguished by considering the number of alternations in the reduced prefix.

Definition 3.4. For $n>0$, a $\Sigma_{n}^{1}$-prefix is one whose reduced prefix begins with $\exists^{1}$ and has $n-1$ alternations of quantifiers. A $\Sigma_{0}^{1}$-prefix is one whose reduced prefix is empty. For $n>0$, a $\Pi_{n}^{1}$-prefix is one whose reduced prefix begins with $\forall^{1}$ and has $n-1$ alternations of quantifiers. A $\Pi_{0}^{1}$-prefix is one whose reduced prefix is empty.

A predicate form is a $\Sigma_{n}^{1}\left(\Pi_{n}^{1}\right)$-form if it has a $\Sigma_{n}^{1}\left(\Pi_{n}^{1}\right)$-prefix. The class of sets in some $\mathbb{N}^{l}$ which can be expressed in $\Sigma_{n}^{1}$-form (respectively, $\Pi_{n}^{1}$-form) is denoted by $\Sigma_{n}^{1}$ (respectively, $\Pi_{n}^{1}$ ).

The class $\Sigma_{0}^{1}=\Pi_{0}^{1}$ is the class of arithmetical sets.

We now recall some well known results about the analytical hierarchy.

Proposition 3.5. Let $R \subseteq \mathbb{N}^{l}$ for some integer $l$. Then $R$ is an analytical set iff there is some integer $n \geq 0$ such that $R \in \Sigma_{n}^{1}$ or $R \in \Pi_{n}^{1}$.

Theorem 3.6. For each integer $n \geq 1$,

(a) $\Sigma_{n}^{1} \cup \Pi_{n}^{1} \subsetneq \Sigma_{n+1}^{1} \cap \Pi_{n+1}^{1}$.

(b) A set $R \subseteq \mathbb{N}^{l}$ is in the class $\Sigma_{n}^{1}$ iff its complement is in the class $\Pi_{n}^{1}$.

(c) $\Sigma_{n}^{1}-\Pi_{n}^{1} \neq \emptyset$ and $\Pi_{n}^{1}-\Sigma_{n}^{1} \neq \emptyset$.

Transformations of prefixes are often used, following the rules given by the next theorem.

Theorem 3.7. For any predicate form with the given prefix, an equivalent predicate form with the new one can be obtained, following the allowed prefix transformations given below :

(a) $\ldots \exists^{0} \exists^{0} \ldots \rightarrow \ldots \exists^{0} \ldots, \quad \ldots \forall^{0} \forall^{0} \ldots \rightarrow \ldots \forall^{0} \ldots$; 
(b) $\ldots \exists^{1} \exists^{1} \ldots \rightarrow \ldots \exists^{1} \ldots, \quad \ldots \forall^{1} \forall^{1} \ldots \rightarrow \ldots \forall^{1} \ldots$;

(c) $\ldots \exists^{0} \quad \ldots \rightarrow \ldots \exists^{1} \ldots, \quad \ldots \forall^{0} \quad \ldots \rightarrow \ldots \forall^{1} \ldots$;

(d) $\ldots \exists^{0} \forall^{1} \ldots \rightarrow \ldots \forall^{1} \exists^{0} \ldots, \quad \ldots \forall^{0} \exists^{1} \ldots \rightarrow \ldots \exists^{1} \forall^{0} \ldots$;

We can now define the notion of 1-reduction and of $\Sigma_{n}^{1}$-complete (respectively, $\Pi_{n}^{1}$-complete) sets. Notice that we give the definition for subsets of $\mathbb{N}$ but one can easily extend this definition to the case of subsets of $\mathbb{N}^{l}$ for some integer $l$.

Definition 3.8. Given two sets $A, B \subseteq \mathbb{N}$ we say A is 1-reducible to $\mathrm{B}$ and write $A \leq_{1} B$ if there exists a total computable injective function $\mathrm{f}$ from $\mathbb{N}$ to $\mathbb{N}$ such that $A=f^{-1}[B]$.

Definition 3.9. A set $A \subseteq \mathbb{N}$ is said to be $\Sigma_{n}^{1}$-complete (respectively, $\Pi_{n}^{1}$-complete) iff $A$ is a $\Sigma_{n}^{1}$-set (respectively, $\Pi_{n}^{1}$-set) and for each $\Sigma_{n}^{1}$-set (respectively, $\Pi_{n}^{1}$-set) $B \subseteq \mathbb{N}$ it holds that $B \leq_{1} A$.

For each integer $n \geq 1$ there exist some $\Sigma_{n}^{1}$-complete set $E_{n} \subseteq \mathbb{N}$. The complement $E_{n}^{-}=\mathbb{N}-E_{n}$ is a $\Pi_{n}^{1}$-complete set. These sets are precisely defined in [21] or [3].

\section{Borel Hierarchy and Analytic Sets}

We assume now the reader to be familiar with basic notions of topology which may be found in [18, 17 , [15, 23, 19].

There is a natural metric on the set $\Sigma^{\omega}$ of infinite words over a finite alphabet $\Sigma$ containing at least two letters which is called the prefix metric and defined as follows. For $u, v \in \Sigma^{\omega}$ and $u \neq v$ let $\delta(u, v)=2^{-l_{\operatorname{pref}(u, v)}}$ where $l_{\operatorname{pref}(u, v)}$ is the first integer $n$ such that the $(n+1)^{s t}$ letter of $u$ is different from the $(n+1)^{s t}$ letter of $v$. This metric induces on $\Sigma^{\omega}$ the usual Cantor topology for which open subsets of $\Sigma^{\omega}$ are in the form $W \cdot \Sigma^{\omega}$, where $W \subseteq \Sigma^{\star}$. A set $L \subseteq \Sigma^{\omega}$ is a closed set iff its complement $\Sigma^{\omega}-L$ is an open set. Define now the Borel Hierarchy of subsets of $\Sigma^{\omega}$ :

Definition 4.1. For a non-null countable ordinal $\alpha$, the classes $\Sigma_{\alpha}^{0}$ and $\Pi_{\alpha}^{0}$ of the Borel Hierarchy on the topological space $\Sigma^{\omega}$ are defined as follows:

$\Sigma_{1}^{0}$ is the class of open subsets of $\Sigma^{\omega}, \Pi_{1}^{0}$ is the class of closed subsets of $\Sigma^{\omega}$,

and for any countable ordinal $\alpha \geq 2$ :

$\boldsymbol{\Sigma}_{\alpha}^{0}$ is the class of countable unions of subsets of $\Sigma^{\omega}$ in $\bigcup_{\gamma<\alpha} \Pi_{\gamma}^{0}$.

$\Pi_{\alpha}^{0}$ is the class of countable intersections of subsets of $\Sigma^{\omega}$ in $\bigcup_{\gamma<\alpha} \Sigma_{\gamma}^{0}$.

For a countable ordinal $\alpha$, a subset of $\Sigma^{\omega}$ is a Borel set of rank $\alpha$ iff it is in $\Sigma_{\alpha}^{0} \cup \Pi_{\alpha}^{0}$ but not in $\bigcup_{\gamma<\alpha}\left(\boldsymbol{\Sigma}_{\gamma}^{0} \cup \boldsymbol{\Pi}_{\gamma}^{0}\right)$

There are also some subsets of $\Sigma^{\omega}$ which are not Borel. Indeed there exists another hierarchy beyond the Borel hierarchy, which is called the projective hierarchy and which is obtained from the Borel hierarchy by successive applications of operations of projection and complementation. The first level of the projective hierarchy is formed by the class of analytic sets and the class of co-analytic sets which are complements of analytic sets. In particular the class of Borel subsets of $\Sigma^{\omega}$ is strictly included into the class $\Sigma_{1}^{1}$ of analytic sets which are obtained by projection of Borel sets. 
Definition 4.2. A subset $A$ of $\Sigma^{\omega}$ is in the class $\Sigma_{1}^{1}$ of analytic sets iff there exists another finite set $Y$ and a Borel subset $B$ of $(\Sigma \times Y)^{\omega}$ such that $x \in A \leftrightarrow \exists y \in Y^{\omega}$ such that $(x, y) \in B$, where $(x, y)$ is the infinite word over the alphabet $\Sigma \times Y$ such that $(x, y)(i)=(x(i), y(i))$ for each integer $i \geq 1$.

We now define completeness with regard to reduction by continuous functions. For a countable ordinal $\alpha \geq 1$, a set $F \subseteq \Sigma^{\omega}$ is said to be a $\Sigma_{\alpha}^{0}$ (respectively, $\Pi_{\alpha}^{0}, \Sigma_{1}^{1}$ )-complete set iff for any set $E \subseteq Y^{\omega}$ (with $Y$ a finite alphabet): $E \in \boldsymbol{\Sigma}_{\alpha}^{0}$ (respectively, $E \in \boldsymbol{\Pi}_{\alpha}^{0}, E \in \boldsymbol{\Sigma}_{1}^{1}$ ) iff there exists a continuous function $f: Y^{\omega} \rightarrow \Sigma^{\omega}$ such that $E=f^{-1}(F) . \Sigma_{n}^{0}$ (respectively $\Pi_{n}^{0}$ )-complete sets, with $n$ an integer $\geq 1$, are thoroughly characterized in [22].

In particular $\mathcal{R}=\left(0^{\star} .1\right)^{\omega}$ is a well known example of $\Pi_{2}^{0}$-complete subset of $\{0,1\}^{\omega}$. It is the set of $\omega$-words over $\{0,1\}$ having infinitely many occurrences of the letter 1 . Its complement $\{0,1\}^{\omega}-\left(0^{\star} .1\right)^{\omega}$ is a $\boldsymbol{\Sigma}_{2}^{0}$-complete subset of $\{0,1\}^{\omega}$.

For $\Gamma$ a finite alphabet having at least two letters, the set $\Gamma^{\omega \times \omega}$ of functions from $\omega \times \omega$ into $\Gamma$ is usually equipped with the product topology of the discrete topology on $\Gamma$. This topology may be defined by the following distance $d$. Let $x$ and $y$ in $\Gamma^{\omega \times \omega}$ such that $x \neq y$, then

$$
\begin{gathered}
d(x, y)=\frac{1}{2^{n}} \quad \text { where } \\
n=\min \{p \geq 0 \mid \exists(i, j) \quad x(i, j) \neq y(i, j) \text { and } i+j=p\} .
\end{gathered}
$$

Then the topological space $\Gamma^{\omega \times \omega}$ is homeomorphic to the topological space $\Gamma^{\omega}$, equipped with the Cantor topology. Borel subsets of $\Gamma^{\omega \times \omega}$ are defined from open subsets as in the case of the topological space $\Gamma^{\omega}$. Analytic subsets of $\Gamma^{\omega \times \omega}$ are obtained as projections on $\Gamma^{\omega \times \omega}$ of Borel subsets of the product space $\Gamma^{\omega \times \omega} \times \Gamma^{\omega}$.

The set $\Sigma^{\omega, \omega}$ of $\omega$-pictures over $\Sigma$, viewed as a topological subspace of $\hat{\Sigma}^{\omega \times \omega}$, is easily seen to be homeomorphic to the topological space $\Sigma^{\omega \times \omega}$, via the mapping $\varphi: \Sigma^{\omega, \omega} \rightarrow \Sigma^{\omega \times \omega}$ defined by $\varphi(p)(i, j)=$ $p(i+1, j+1)$ for all $p \in \Sigma^{\omega, \omega}$ and $i, j \in \omega$.

\section{Highly Undecidable Problems}

We are now going to study decision problems about recognizable languages of infinite pictures. We shall use some results of Castro and Cucker who studied degrees of decision problems for $\omega$-languages accepted by Turing machines and proved that many of them are highly undecidable, [3].

So we now recall the notion of acceptance of infinite words by Turing machines considered by Castro and Cucker in [3].

Definition 5.1. A non deterministic Turing machine $\mathcal{M}$ is a 5 -tuple $\mathcal{M}=\left(Q, \Sigma, \Gamma, \delta, q_{0}\right)$, where $Q$ is a finite set of states, $\Sigma$ is a finite input alphabet, $\Gamma$ is a finite tape alphabet satisfying $\Sigma \subseteq \Gamma, q_{0}$ is the initial state, and $\delta$ is a mapping from $Q \times \Gamma$ to subsets of $Q \times \Gamma \times\{L, R, S\}$. A configuration of $\mathcal{M}$ is a 3-tuple $(q, \sigma, i)$, where $q \in Q, \sigma \in \Gamma^{\omega}$ and $i \in \mathbb{N}$. An infinite sequence of configurations $r=\left(q_{i}, \alpha_{i}, j_{i}\right)_{i \geq 1}$ is called a run of $\mathcal{M}$ on $w \in \Sigma^{\omega}$ iff:

(a) $\left(q_{1}, \alpha_{1}, j_{1}\right)=\left(q_{0}, w, 1\right)$, and 
(b) for each $i \geq 1,\left(q_{i}, \alpha_{i}, j_{i}\right) \vdash\left(q_{i+1}, \alpha_{i+1}, j_{i+1}\right)$,

where $\vdash$ is the transition relation of $\mathcal{M}$ defined as usual. The run $r$ is said to be complete if the limsup of the head positions is infinity, i.e. if $(\forall n \geq 1)(\exists k \geq 1)\left(j_{k} \geq n\right)$. The run $r$ is said to be oscillating if the liminf of the head positions is bounded, i.e. if $(\exists k \geq 1)(\forall n \geq 1)(\exists m \geq n)\left(j_{m}=k\right)$.

Definition 5.2. Let $\mathcal{M}=\left(Q, \Sigma, \Gamma, \delta, q_{0}\right)$ be a non deterministic Turing machine and $F \subseteq Q$. The $\omega$-language accepted by $(\mathcal{M}, F)$ is the set of $\omega$-words $\sigma \in \Sigma^{\omega}$ such that there exists a complete non oscillating run $r=\left(q_{i}, \alpha_{i}, j_{i}\right)_{i \geq 1}$ of $\mathcal{M}$ on $\sigma$ such that, for all $i, q_{i} \in F$.

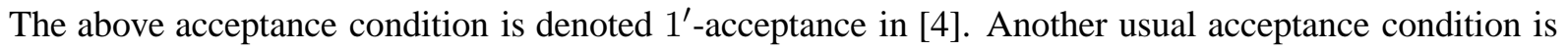
the now called Büchi acceptance condition which is also denoted 2-acceptance in [卂]. We now recall its definition.

Definition 5.3. Let $\mathcal{M}=\left(Q, \Sigma, \Gamma, \delta, q_{0}\right)$ be a non deterministic Turing machine and $F \subseteq Q$. The $\omega$ language Büchi accepted by $(\mathcal{M}, F)$ is the set of $\omega$-words $\sigma \in \Sigma^{\omega}$ such that there exists a complete non oscillating run $r=\left(q_{i}, \alpha_{i}, j_{i}\right)_{i \geq 1}$ of $\mathcal{M}$ on $\sigma$ and infinitely many integers $i$ such that $q_{i} \in F$.

Recall that Cohen and Gold proved in [曰, Theorem 8.6] that one can effectively construct, from a given non deterministic Turing machine, another equivalent non deterministic Turing machine, equipped with the same kind of acceptance condition, and in which every run is complete non oscillating. Cohen and Gold proved also in [ [ 4 , Theorem 8.2] that an $\omega$-language is accepted by a non deterministic Turing machine with $1^{\prime}$-acceptance condition iff it is accepted by a non deterministic Turing machine with Büchi acceptance condition.

From now on, we shall denote $\mathcal{M}_{z}$ the non deterministic Turing machine of index $z$, (accepting words over $\Sigma=\{a, b\}$ ), equipped with a $1^{\prime}$-acceptance condition. In a similar way we shall denote $\mathcal{T}_{z}$ the non deterministic tiling system of index $z$, (accepting pictures over $\Sigma=\{a, b\}$ ), equipped with a Büchi acceptance condition.

For $\sigma \in \Sigma^{\omega}=\{a, b\}^{\omega}$ we denote $\sigma^{a}$ the $\omega$-picture whose first row is the $\omega$-word $\sigma$ and whose other rows are labelled with the letter $a$. For an $\omega$-language $L \subseteq \Sigma^{\omega}=\{a, b\}^{\omega}$ we denote $L^{a}$ the language of infinite pictures $\left\{\sigma^{a} \mid \sigma \in L\right\}$.

We can now recall a result proved in [9] which will be useful later.

\section{Lemma 5.4. ([阿)}

If $L \subseteq \Sigma^{\omega}$ is accepted by some Turing machine (in which every run is complete non oscillating) with a Büchi acceptance condition, then $L^{a}$ is Büchi recognizable by a finite tiling system.

Proof. Let $L \subseteq \Sigma^{\omega}$ be an $\omega$-language accepted by some Turing machine $\mathcal{M}=\left(Q, \Sigma, \Gamma, \delta, q_{0}\right)$ with a Büchi acceptance condition, where $F \subseteq Q$ is the set of accepting states.

We assume that the Turing machine has a single semi-infinite tape, with one reading head which may also write on the tape.

Cohen and Gold proved that one can consider only such a restricted model of Turing machines [ $₫$ ].

An instantaneous configuration of $\mathcal{M}$ is given by an infinite word $u . q . v$ where $u \in \Gamma^{\star}, q \in Q, v \in \Gamma^{\omega}$, 
and the first letter of $v$ is the one scanned by the head of $\mathcal{M}$.

The initial configuration of $\mathcal{M}$ reading the infinite word $\sigma \in \Sigma^{\omega}$ is $q_{0} . \sigma$.

A computation of $\mathcal{M}$ reading $\sigma \in \Sigma^{\omega}$ is an infinite sequence of configurations $\alpha_{0}, \alpha_{1}, \alpha_{2}, \ldots, \alpha_{i}, \ldots$, where $\alpha_{0}=q_{0} \cdot \sigma$ is the initial configuration and for all integers $i \geq 0, \alpha_{i}=u_{i} \cdot q_{i} \cdot v_{i}$ is the $(i+1)^{t h}$ configuration.

The computation is successful if and only if there exists a final state $q_{f} \in F$ and infinitely many integers $i$ such that $q_{i}=q_{f}$.

Using a similar reasoning as in the classical proof of the undecidability of the emptiness problem for recognizable languages of finite pictures, [11, p. 34], we can define a set of tiles $\Delta$ in such a way that for $\sigma \in \Sigma^{\omega}$, a run $\rho$ of the tiling system $\mathcal{T}=(\Sigma, \Gamma \cup Q, \Delta, F)$ over the infinite picture $\sigma^{a}$ satifies:

$$
\text { for each integer } i \geq 0 \quad \rho(0, i) \cdot \rho(1, i) \cdot \rho(2, i) \ldots=\alpha_{i}=u_{i} \cdot q_{i} \cdot v_{i}
$$

i.e. $\rho(0, i) \cdot \rho(1, i) \cdot \rho(2, i) \ldots$ is the $(i+1)^{t h}$ configuration of $\mathcal{M}$ reading the $\omega$-word $\sigma \in \Sigma^{\omega}$. Thus the Büchi tiling system $(\mathcal{T}, F)$ recognizes the language $L^{a}$.

Notice that the above cited constructions of [4] and of the proof of Lemma 5.4 are effective and that they can be achieved in an injective way. This is expressed by the following lemma.

Lemma 5.5. There is an injective computable function $K$ from $\mathbb{N}$ into $\mathbb{N}$ satisfying the following property.

If $\mathcal{M}_{z}$ is the non deterministic Turing machine (equipped with a $1^{\prime}$-acceptance condition) of index $z$, and if $\mathcal{T}_{K(z)}$ is the tiling system (equipped with a Büchi acceptance condition) of index $K(z)$, then

$$
L\left(\mathcal{M}_{z}\right)^{a}=L^{B}\left(\mathcal{T}_{K(z)}\right)
$$

Recall that Castro and Cucker proved in [3] that the non-emptiness problem and the infiniteness problem for $\omega$-languages of Turing machines are both $\Sigma_{1}^{1}$-complete. We can now easily infer from Lemma $5.5 \mathrm{a}$ similar result for recognizable languages of infinite pictures.

Theorem 5.6. The non-emptiness problem and the infiniteness problem for Büchi-recognizable languages of infinite pictures are $\Sigma_{1}^{1}$-complete, i.e. :

1. $\left\{z \in \mathbb{N} \mid L^{B}\left(\mathcal{T}_{z}\right) \neq \emptyset\right\}$ is $\Sigma_{1}^{1}$-complete.

2. $\left\{z \in \mathbb{N} \mid L^{B}\left(\mathcal{T}_{z}\right)\right.$ is infinite $\}$ is $\Sigma_{1}^{1}$-complete.

Proof. We first show that these two decision problems are in the class $\Sigma_{1}^{1}$.

Notice first that, using a recursive bijection $b:(\mathbb{N}-\{0\})^{2} \rightarrow \mathbb{N}-\{0\}$, one can associate to each $\omega$-word $\sigma \in \Sigma^{\omega}$ a unique $\omega$-picture $p^{\sigma} \in \Sigma^{\omega, \omega}$ which is simply defined by $p^{\sigma}(i, j)=\sigma(b(i, j))$ for all integers $i, j \geq 1$.

On the other hand a run of a tiling system $\mathcal{A}=(Q, \Sigma, \Delta)$ over an $\omega$-picture $p \in \Sigma^{\omega, \omega}$ is a mapping $\rho$ from $\omega \times \omega$ into $Q$, i.e. an element of $Q^{\omega \times \omega}$. Using again a recursive bijection between $(\mathbb{N})^{2}$ and $\mathbb{N}$, we can identify a run $\rho$ with an element of $Q^{\omega}$ and finally with a coding of this element over the alphabet $\{0,1\}$. So the run $\rho$ can be identified with its code $\bar{\rho} \in\{0,1\}^{\omega}$. 
Assume now that the tiling system $\mathcal{A}=(Q, \Sigma, \Delta)$ is equipped with a set of accepting states $F \subseteq Q$. It is then easy to see that for $\sigma \in \Sigma^{\omega}$ and $\rho \in\{0,1\}^{\omega}$, " $\rho$ is a Büchi accepting run of $(\mathcal{A}, F)$ over the $\omega$-picture $p^{\sigma}$ " can be expressed by an arithmetical formula, see also [1], Section 2.4].

We can now express " $L^{B}\left(\mathcal{T}_{z}\right) \neq \emptyset$ ” by " $\exists \sigma \in \Sigma^{\omega} \exists \rho \in\{0,1\}^{\omega} \quad$ [ $\rho$ is a Büchi accepting run of $\mathcal{T}_{z}$ over the $\omega$-picture $p^{\sigma}$ ]" which is a $\Sigma_{1}^{1}$-formula.

In order to show that " $L^{B}\left(\mathcal{T}_{z}\right)$ is infinite" can be also expressed by a $\Sigma_{1}^{1}$-formula, we shall use again the bijection $b:(\mathbb{N}-\{0\})^{2} \rightarrow \mathbb{N}-\{0\}$.

We can consider an infinite word $\sigma \in \Sigma^{\omega}$ as a countably infinite family of infinite words over $\Sigma$ : the family of $\omega$-words $\left(\sigma_{i}\right)$ such that for each $i \geq 1, \sigma_{i}$ is defined by $\sigma_{i}(j)=\sigma(b(i, j))$ for each $j \geq 1$. In a similar manner an $\omega$-word $\rho \in\{0,1\}^{\omega}$ can be considered as a countably infinite family of infinite words $\left(\rho_{i}\right)$ defined, for each $i \geq 1$, by $\rho_{i}(j)=\rho(b(i, j))$ for each $j \geq 1$.

We can now express " $L^{B}\left(\mathcal{T}_{z}\right)$ is infinite" by the formula " $\exists \sigma \in \Sigma^{\omega} \quad \exists \rho \in\{0,1\}^{\omega} \quad$ [ ( all $\omega$-words $\sigma_{i}$ are distinct ) and (for each integer $i \geq 1, \rho_{i}$ is a Büchi accepting run of $\mathcal{T}_{z}$ over the $\omega$-picture $p^{\sigma_{i}}$ )]" . This is a $\Sigma_{1}^{1}$-formula because "all $\omega$-words $\sigma_{i}$ are distinct" can be expressed by the arithmetical formula: " $(\forall j>k \geq 1)(\exists i \geq 1) \sigma_{j}(i) \neq \sigma_{k}(i) "$.

Using the reduction $K$ given by Lemma 5.5 we can easily see that $L\left(\mathcal{M}_{z}\right)$ is empty (respectively, infinite) if and only if $L^{B}\left(\mathcal{T}_{K(z)}\right)=L\left(\mathcal{M}_{z}\right)^{a}$ is empty (respectively, infinite). This proves that

$$
\begin{gathered}
\left\{z \in \mathbb{N} \mid L\left(\mathcal{M}_{z}\right) \neq \emptyset\right\} \leq_{1}\left\{z \in \mathbb{N} \mid L^{B}\left(\mathcal{T}_{z}\right) \neq \emptyset\right\} \\
\left\{z \in \mathbb{N} \mid L\left(\mathcal{M}_{z}\right) \text { is infinite }\right\} \leq_{1}\left\{z \in \mathbb{N} \mid L^{B}\left(\mathcal{T}_{z}\right) \text { is infinite }\right\}
\end{gathered}
$$

and then the completeness result follows from the $\Sigma_{1}^{1}$-completeness of the non-emptiness problem and of the infiniteness problem for $\omega$-languages of Turing machines.

On the other hand it is easy to see that the language $\Sigma^{\omega, \omega}-\left(\Sigma^{\omega}\right)^{a}$ of $\omega$-pictures is Büchi recognizable. But the class $T S\left(\Sigma^{\omega, \omega}\right)$ is closed under finite union, so we get the following result.

Lemma 5.7. If $L \subseteq \Sigma^{\omega}$ is accepted by some Turing machine with a Büchi acceptance condition, then $L^{a} \cup\left[\Sigma^{\omega, \omega}-\left(\Sigma^{\omega}\right)^{a}\right]$ is Büchi recognizable by a finite tiling system.

Notice that the constructions are effective and that they can be achieved in an injective way, so we can now state the following lemma, asserting the existence of a computable function $H$ which will be often used in the sequel.

Lemma 5.8. There is an injective computable function $H$ from $\mathbb{N}$ into $\mathbb{N}$ satisfying the following property.

If $\mathcal{M}_{z}$ is the non deterministic Turing machine (equipped with a $1^{\prime}$-acceptance condition) of index $z$, and if $\mathcal{T}_{H(z)}$ is the tiling system (equipped with a Büchi acceptance condition) of index $H(z)$, then

$$
L\left(\mathcal{M}_{z}\right)^{a} \cup\left[\Sigma^{\omega, \omega}-\left(\Sigma^{\omega}\right)^{a}\right]=L^{B}\left(\mathcal{T}_{H(z)}\right)
$$


We can now prove that the universality problem for Büchi-recognizable languages of infinite pictures is highly undecidable and give its exact degree.

Theorem 5.9. The universality problem for Büchi-recognizable languages of infinite pictures is $\Pi_{2}^{1}$ complete, i.e. : $\quad\left\{z \in \mathbb{N} \mid L^{B}\left(\mathcal{T}_{z}\right)=\Sigma^{\omega, \omega}\right\}$ is $\Pi_{2}^{1}$-complete.

Proof. We first check that the set $\left\{z \in \mathbb{N} \mid L^{B}\left(\mathcal{T}_{z}\right)=\Sigma^{\omega, \omega}\right\}$ is in the class $\Pi_{2}^{1}$. We can write that $L^{B}\left(\mathcal{T}_{z}\right)=\Sigma^{\omega, \omega}$ if and only if " $\forall \sigma \in \Sigma^{\omega} \exists \rho \in\{0,1\}^{\omega}$ ( $\rho$ is a Büchi-accepting run of $\mathcal{T}_{z}$ over $p^{\sigma}$ )". The two quantifiers of type 1 are followed by an arithmetical formula. Thus the set $\left\{z \in \mathbb{N} \mid L^{B}\left(\mathcal{T}_{z}\right)=\Sigma^{\omega, \omega}\right\}$ is in the class $\Pi_{2}^{1}$.

In order to prove completeness we use the corresponding result for Turing machines proved in [3]: the set $\left\{z \in \mathbb{N} \mid L\left(\mathcal{M}_{z}\right)=\Sigma^{\omega}\right\}$ is $\Pi_{2}^{1}$-complete. Consider now the injective computable function $H$ from $\mathbb{N}$ into $\mathbb{N}$ given in Lemma 5.8. It is easy to see that for any Turing machine $\mathcal{M}_{z}$ it holds that $L\left(\mathcal{M}_{z}\right)=\Sigma^{\omega}$ if and only if $L\left(\mathcal{M}_{z}\right)^{a} \cup\left[\Sigma^{\omega, \omega}-\left(\Sigma^{\omega}\right)^{a}\right]=L^{B}\left(\mathcal{T}_{H(z)}\right)=\Sigma^{\omega, \omega}$. This proves that $\left\{z \in \mathbb{N} \mid L\left(\mathcal{M}_{z}\right)=\Sigma^{\omega}\right\} \leq_{1}\left\{z \in \mathbb{N} \mid L^{B}\left(\mathcal{T}_{z}\right)=\Sigma^{\omega, \omega}\right\}$, thus this latter set is $\Pi_{2}^{1}$-complete.

We now consider the inclusion and the equivalence problems for Büchi-recognizable languages of infinite pictures.

Theorem 5.10. The inclusion and the equivalence problems for Büchi-recognizable languages of infinite pictures are $\Pi_{2}^{1}$-complete, i.e. :

1. $\left\{(y, z) \in \mathbb{N}^{2} \mid L^{B}\left(\mathcal{T}_{y}\right) \subseteq L^{B}\left(\mathcal{T}_{z}\right)\right\}$ is $\Pi_{2}^{1}$-complete.

2. $\left\{(y, z) \in \mathbb{N}^{2} \mid L^{B}\left(\mathcal{T}_{y}\right)=L^{B}\left(\mathcal{T}_{z}\right)\right\}$ is $\Pi_{2}^{1}$-complete.

Proof. We first prove that the set $\left\{(y, z) \in \mathbb{N}^{2} \mid L^{B}\left(\mathcal{T}_{y}\right) \subseteq L^{B}\left(\mathcal{T}_{z}\right)\right\}$ is a $\Pi_{2}^{1}$-set. It suffices to remark that " $L^{B}\left(\mathcal{T}_{y}\right) \subseteq L^{B}\left(\mathcal{T}_{z}\right)$ " can be expressed by the $\Pi_{2}^{1}$-formula : " $\forall \sigma \in \Sigma^{\omega} \forall \rho \in\{0,1\}^{\omega} \exists \rho^{\prime} \in\{0,1\}^{\omega}$ [if ( $\rho$ is a Büchi accepting run of $\mathcal{T}_{y}$ over $\left.p^{\sigma}\right)$, then $\left(\rho^{\prime}\right.$ is a Büchi accepting run of $\mathcal{T}_{z}$ over $\left.p^{\sigma}\right)$ ]".

Then the set $\left\{(y, z) \in \mathbb{N}^{2} \mid L^{B}\left(\mathcal{T}_{y}\right)=L^{B}\left(\mathcal{T}_{z}\right)\right\}$ which is the intersection of the two sets $\left\{(y, z) \in \mathbb{N}^{2} \mid\right.$ $\left.L^{B}\left(\mathcal{T}_{y}\right) \subseteq L^{B}\left(\mathcal{T}_{z}\right)\right\}$ and $\left\{(y, z) \in \mathbb{N}^{2} \mid L^{B}\left(\mathcal{T}_{z}\right) \subseteq L^{B}\left(\mathcal{T}_{y}\right)\right\}$ is also a $\Pi_{2}^{1}$-set.

On the other hand it is easy to check that for all integers $y, z$, it holds that $L\left(\mathcal{M}_{y}\right) \subseteq L\left(\mathcal{M}_{z}\right)$ iff $L^{B}\left(\mathcal{T}_{H(y)}\right) \subseteq L^{B}\left(\mathcal{T}_{H(z)}\right)$ and that $L\left(\mathcal{M}_{y}\right)=L\left(\mathcal{M}_{z}\right)$ iff $L^{B}\left(\mathcal{T}_{H(y)}\right)=L^{B}\left(\mathcal{T}_{H(z)}\right)$. Thus using the reduction $H$ we see that

$$
\begin{aligned}
& \left\{(y, z) \in \mathbb{N}^{2} \mid L\left(\mathcal{M}_{y}\right) \subseteq L\left(\mathcal{M}_{z}\right)\right\} \leq_{1}\left\{(y, z) \in \mathbb{N}^{2} \mid L^{B}\left(\mathcal{T}_{y}\right) \subseteq L^{B}\left(\mathcal{T}_{z}\right)\right\} \\
& \left\{(y, z) \in \mathbb{N}^{2} \mid L\left(\mathcal{M}_{y}\right)=L\left(\mathcal{M}_{z}\right)\right\} \leq_{1}\left\{(y, z) \in \mathbb{N}^{2} \mid L^{B}\left(\mathcal{T}_{y}\right)=L^{B}\left(\mathcal{T}_{z}\right)\right\}
\end{aligned}
$$

The $\Pi_{2}^{1}$-completeness follows then from the $\Pi_{2}^{1}$-completeness of the inclusion and the equivalence problems for $\omega$-languages of Turing machines proved in [3].

We are going to consider now the decision problems studied in [9]. Using topological arguments, we gave in [9] the answer to two questions raised in [1], showing that it is undecidable whether a Büchi 
recognizable language of infinite pictures is E-recognizable (respectively, A-recognizable). We are going to show that these problems are actually $\Pi_{2}^{1}$-complete, using again some topological arguments.

Theorem 5.11. The problem to determine whether a given Büchi-recognizable language of infinite pictures is E-recognizable (respectively, A-recognizable) is $\Pi_{2}^{1}$-complete, i.e. :

1. $\left\{z \in \mathbb{N} \mid L^{B}\left(\mathcal{T}_{z}\right)\right.$ is E-recognizable $\}$ is $\Pi_{2}^{1}$-complete.

2. $\left\{z \in \mathbb{N} \mid L^{B}\left(\mathcal{T}_{z}\right)\right.$ is A-recognizable $\}$ is $\Pi_{2}^{1}$-complete.

Proof. We first prove that the set $\left\{z \in \mathbb{N} \mid L^{B}\left(\mathcal{T}_{z}\right)\right.$ is E-recognizable $\}$ is a $\Pi_{2}^{1}$-set. The sentence " $L^{B}\left(\mathcal{T}_{z}\right)$ is E-recognizable" can be expressed by " $\exists y \quad L^{B}\left(\mathcal{T}_{z}\right)=L^{E}\left(\mathcal{T}_{y}\right)$ ". The assertion " $L^{B}\left(\mathcal{T}_{z}\right)=$ $L^{E}\left(\mathcal{T}_{y}\right)$ " can be expressed by a $\Pi_{2}^{1}$-formula in a very similar manner as " $L^{B}\left(\mathcal{T}_{z}\right)=L^{B}\left(\mathcal{T}_{y}\right)$ " was (see the proof of Theorem 5.10), because for $\sigma \in \Sigma^{\omega}$ and $\rho \in\{0,1\}^{\omega}$ the sentence " $\rho$ is a E-accepting run of $\mathcal{T}_{z}$ over the $\omega$-picture $p^{\sigma}$ )" can be expressed by an arithmetical formula. Moreover the quantifier $\exists y$ is of type 0 thus " $L^{B}\left(\mathcal{T}_{z}\right)$ is E-recognizable" can be expressed by a $\Pi_{2}^{1}$-formula.

We prove in a very similar manner that " $L^{B}\left(\mathcal{T}_{z}\right)$ is A-recognizable" can be expressed by a $\Pi_{2}^{1}$-formula. Details are here left to the reader.

We now prove the completeness part of the result. We first define a simple operation over $\omega$-languages. For two $\omega$-words $x, x^{\prime} \in \Sigma^{\omega}$ the $\omega$-word $x \otimes x^{\prime}$ is just the shuffle of the two $\omega$-words $x$ and $x^{\prime}$ which is simply defined by : for every integer $n \geq 1\left(x \otimes x^{\prime}\right)(2 n-1)=x(n)$ and $\left(x \otimes x^{\prime}\right)(2 n)=x^{\prime}(n)$. For two $\omega$-languages $L, L^{\prime} \subseteq \Sigma^{\omega}$, the $\omega$-language $L \otimes L^{\prime}$ is defined by $L \otimes L^{\prime}=\left\{x \otimes x^{\prime} \mid x \in L\right.$ and $\left.x^{\prime} \in L^{\prime}\right\}$.

We shall use the following construction. We know that there is a simple example of $\boldsymbol{\Sigma}_{1}^{1}$-complete set $L \subseteq \Sigma^{\omega}$ accepted by a 1-counter automaton, hence by a Turing machine with $1^{\prime}$ acceptance condition, see [8]. Then it is easy to define an injective computable function $\theta$ from $\mathbb{N}$ into $\mathbb{N}$ such that, for every integer $z \in \mathbb{N}$, it holds that $L\left(\mathcal{M}_{\theta(z)}\right)=\left(L \otimes \Sigma^{\omega}\right) \cup\left(\Sigma^{\omega} \otimes L\left(\mathcal{M}_{z}\right)\right)$.

We are going to use now the reduction $H$ already considered above. We have seen that

$$
L\left(\mathcal{M}_{z}\right)=\Sigma^{\omega} \text { if and only if } L\left(\mathcal{T}_{H(z)}\right)=\Sigma^{\omega, \omega}
$$

and we can easily see that

$$
L\left(\mathcal{M}_{\theta(z)}\right)=\Sigma^{\omega} \text { if and only if } L\left(\mathcal{M}_{z}\right)=\Sigma^{\omega}
$$

because $L \neq \Sigma^{\omega}$.

The reduction $H \circ \theta$ is an injective computable function from $\mathbb{N}$ into $\mathbb{N}$.

We consider now two cases.

First case. $L\left(\mathcal{M}_{z}\right)=\Sigma^{\omega}$. Then $L\left(\mathcal{M}_{\theta(z)}\right)=\Sigma^{\omega}$ and $L^{B}\left(\mathcal{T}_{H \circ \theta(z)}\right)=\Sigma^{\omega, \omega}$. In particular $L^{B}\left(\mathcal{T}_{H \circ \theta(z)}\right)$ is E-recognized (respectively, A-recognized) by a tiling system.

Second case. $L\left(\mathcal{M}_{z}\right) \neq \Sigma^{\omega}$. Then there is an $\omega$-word $x \in \Sigma^{\omega}$ such that $x \notin L\left(\mathcal{M}_{z}\right)$. But $L\left(\mathcal{M}_{\theta(z)}\right)=$ $\left(L \otimes \Sigma^{\omega}\right) \cup\left(\Sigma^{\omega} \otimes L\left(\mathcal{M}_{z}\right)\right)$ thus $\left\{\sigma \in \Sigma^{\omega} \mid \sigma \otimes x \in L\left(\mathcal{M}_{\theta(z)}\right)\right\}=L$ is a $\Sigma_{1}^{1}$-complete set. The function $\psi_{x}: \sigma \rightarrow \sigma \otimes x$ is continuous. This implies that $L\left(\mathcal{M}_{\theta(z)}\right)$ is not a Borel set because otherwise 
$L=\left\{\sigma \in \Sigma^{\omega} \mid \sigma \otimes x \in L\left(\mathcal{M}_{\theta(z)}\right)\right\}=\psi_{x}^{-1}\left(L\left(\mathcal{M}_{\theta(z)}\right)\right)$ would be also Borel as the inverse image of a Borel set by a continuous function [15].

Then it is easy to see that $L^{B}\left(\mathcal{T}_{H \circ \theta(z)}\right)=L\left(\mathcal{M}_{\theta(z)}\right)^{a} \cup\left[\Sigma^{\omega, \omega}-\left(\Sigma^{\omega}\right)^{a}\right]$ is not a Borel set. But it was proved in [9, Lemma 5.2] that every E-recognized language of infinite pictures is a $\Sigma_{2}^{0}$-set and in [9, Lemma 5.3] that every A-recognized language of infinite pictures is a closed set.

Thus in that case the $\omega$-picture language $L^{B}\left(\mathcal{T}_{H \circ \theta(z)}\right)$ is neither E-recognizable nor A-recognizable.

Finally, using the reduction $H \circ \theta$, we have proved that :

$$
\begin{aligned}
& \left\{z \in \mathbb{N} \mid L\left(\mathcal{M}_{z}\right)=\Sigma^{\omega}\right\} \leq_{1}\left\{z \in \mathbb{N} \mid L^{B}\left(\mathcal{T}_{z}\right) \text { is E-recognizable }\right\} \\
& \left\{z \in \mathbb{N} \mid L\left(\mathcal{M}_{z}\right)=\Sigma^{\omega}\right\} \leq_{1}\left\{z \in \mathbb{N} \mid L^{B}\left(\mathcal{T}_{z}\right) \text { is A-recognizable }\right\}
\end{aligned}
$$

and this ends the proof.

As in [9] we are going to infer from the proof of (high) undecidability of E-recognizability (respectively, A-recognizability) some other (high) undecidability results.

It was proved in [9] that for any Borel class $\Sigma_{\alpha}^{0}$ or $\Pi_{\alpha}^{0}$, it is undecidable whether a given Büchirecognizable language of $\omega$-pictures is in $\Sigma_{\alpha}^{0}$ (respectively, is in $\Pi_{\alpha}^{0}$, is a Borel set). We can deduce from the above proof that the topological complexity of recognizable languages of infinite pictures is in fact highly undecidable.

Theorem 5.12. Let $\alpha$ be a non-null countable ordinal. Then

1. $\left\{z \in \mathbb{N} \mid L^{B}\left(\mathcal{T}_{z}\right)\right.$ is in the Borel class $\left.\Sigma_{\alpha}^{0}\right\}$ is $\Pi_{2}^{1}$-hard.

2. $\left\{z \in \mathbb{N} \mid L^{B}\left(\mathcal{T}_{z}\right)\right.$ is in the Borel class $\left.\Pi_{\alpha}^{0}\right\}$ is $\Pi_{2}^{1}$-hard.

3. $\left\{z \in \mathbb{N} \mid L^{B}\left(\mathcal{T}_{z}\right)\right.$ is a Borel set $\}$ is $\Pi_{2}^{1}$-hard.

Proof. We can use the same reduction $H \circ \theta$ as in the proof of Theorem 5.11. We have seen that there are two cases.

First case. $L\left(\mathcal{M}_{z}\right)=\Sigma^{\omega}$. Then $L\left(\mathcal{M}_{\theta(z)}\right)=\Sigma^{\omega}$ and $L^{B}\left(\mathcal{T}_{H \circ \theta(z)}\right)=\Sigma^{\omega, \omega}$. In particular $L^{B}\left(\mathcal{T}_{H \circ \theta(z)}\right)=$ $\Sigma^{\omega, \omega}$ is an open and closed subset of $\Sigma^{\omega, \omega}$ and it belongs to all Borel classes $\boldsymbol{\Sigma}_{\alpha}^{0}$ and $\boldsymbol{\Pi}_{\alpha}^{0}$.

Second case. $L\left(\mathcal{M}_{z}\right) \neq \Sigma^{\omega}$. Then we have seen that $L^{B}\left(\mathcal{T}_{H \circ \theta(z)}\right)$ is not a Borel set.

Finally, using the reduction $H \circ \theta$, the result follows from the $\Pi_{2}^{1}$-completeness of the universality problem for $\omega$-languages of Turing machines.

We now come to the complementability problem. The class of Büchi-recognizable languages of infinite pictures is not closed under complement [1]. Thus the question naturally arises: "can we decide whether the complement of a Büchi-recognizable language of infinite pictures is Büchi-recognizable?". It has been proved in [9] that this problem is undecidable. We are going to prove that it is in fact $\Pi_{2}^{1}$-complete.

Another classical problem is the determinizability problem: "can we decide whether a given recognizable language of infinite pictures is recognized by a deterministic tiling system?". Again this problem 
has been proved to be undecidable in [9] and we shall prove it is in fact $\Pi_{2}^{1}$-complete.

Recall that a tiling system is called deterministic if on any picture it allows at most one tile covering the origin, the state assigned to position $(i+1, j+1)$ is uniquely determined by the states at positions $(i, j),(i+1, j),(i, j+1)$ and the states at the border positions $(0, j+1)$ and $(i+1,0)$ are determined by the state $(0, j)$, respectively $(i, 0)$, [1].

As remarked in [1], the hierarchy proofs of the classical Landweber hierarchy defined using deterministic $\omega$-automata "carry over without essential changes to pictures". In particular it is easy to see that a language of $\omega$-pictures which is Büchi-recognized by a deterministic tiling system is a $\Pi_{2}^{0}$-set and that a language of $\omega$-pictures which is Muller-recognized by a deterministic tiling system is a boolean combination of $\Pi_{2}^{0}$-sets, hence a $\boldsymbol{\Delta}_{3}^{0}$-set.

We can now state the following results.

Theorem 5.13. The determinizability problem and the complementability problem for Büchi-recognizable languages of infinite pictures are $\Pi_{2}^{1}$-complete, i.e. :

1. $\left\{z \in \mathbb{N} \mid L^{B}\left(\mathcal{T}_{z}\right)\right.$ is Büchi-recognizable by a deterministic tiling system $\}$ is $\Pi_{2}^{1}$-complete.

2. $\left\{z \in \mathbb{N} \mid L^{B}\left(\mathcal{T}_{z}\right)\right.$ is Muller-recognizable by a deterministic tiling system $\}$ is $\Pi_{2}^{1}$-complete.

3. $\left\{z \in \mathbb{N} \mid \exists y \Sigma^{\omega, \omega}-L^{B}\left(\mathcal{T}_{z}\right)=L^{B}\left(\mathcal{T}_{y}\right)\right\}$ is $\Pi_{2}^{1}$-complete.

Proof. It is easy to see that the set $D$ of indices of deterministic tiling systems equipped with a Büchi acceptance condition is recursive. The formula $\exists y \in D \quad L^{B}\left(\mathcal{T}_{z}\right)=L^{B}\left(\mathcal{T}_{y}\right)$ can be written : " $\exists y[y \in$ $D$ and $L^{B}\left(\mathcal{T}_{z}\right)=L^{B}\left(\mathcal{T}_{y}\right)$ ]" and it can be expressed by a $\Pi_{2}^{1}$-formula because the quantifier $\exists y$ is of type 0 and " $L^{B}\left(\mathcal{T}_{z}\right)=L^{B}\left(\mathcal{T}_{y}\right)$ " can be expressed by a $\Pi_{2}^{1}$-formula. Thus the set $\left\{z \in \mathbb{N} \mid \exists y \in D L^{B}\left(\mathcal{T}_{z}\right)=\right.$ $\left.L^{B}\left(\mathcal{T}_{y}\right)\right\}$ is in the class $\Pi_{2}^{1}$.

The case of deterministic tiling systems with Muller acceptance condition is very similar. Details are here left to the reader.

On the other hand " $\Sigma^{\omega, \omega}-L^{B}\left(\mathcal{T}_{z}\right)=L^{B}\left(\mathcal{T}_{y}\right)$ " can be expressed by a $\Pi_{2}^{1}$-formula so " $\exists y \quad \Sigma^{\omega, \omega}-$ $L^{B}\left(\mathcal{T}_{z}\right)=L^{B}\left(\mathcal{T}_{y}\right)$ " can be expressed by a $\Pi_{2}^{1}$-formula because the quantifier $\exists y$ is of type 0 . Thus the set $\left\{z \in \mathbb{N} \mid \exists y \Sigma^{\omega, \omega}-L^{B}\left(\mathcal{T}_{z}\right)=L^{B}\left(\mathcal{T}_{y}\right)\right\}$ is in the class $\Pi_{2}^{1}$.

To prove completeness, we use the same reduction $H \circ \theta$ as in the proof of Theorem 5.11. We have seen that there are two cases.

First case. $L\left(\mathcal{M}_{z}\right)=\Sigma^{\omega}$ and then $L^{B}\left(\mathcal{T}_{H \circ \theta(z)}\right)=\Sigma^{\omega, \omega}$. In particular $L^{B}\left(\mathcal{T}_{H \circ \theta(z)}\right)=\Sigma^{\omega, \omega}$ is accepted by a Büchi deterministic tiling system and also by a Muller deterministic tiling system. Morever its complement is empty so it is Büchi (or Muller) recognized by a tiling system.

Second case. $L\left(\mathcal{M}_{z}\right) \neq \Sigma^{\omega}$. Then we have seen that $L^{B}\left(\mathcal{T}_{H \circ \theta(z)}\right)$ is not a Borel set. Thus in that case $L^{B}\left(\mathcal{T}_{H \circ \theta(z)}\right)$ cannot be accepted by any deterministic tiling system with Büchi or Muller acceptance condition. Moreover its complement $\Sigma^{\omega, \omega}-L^{B}\left(\mathcal{T}_{H \circ \theta(z)}\right)$ is not a $\boldsymbol{\Sigma}_{1}^{1}$-subset of $\Sigma^{\omega, \omega}$ because otherwise $L^{B}\left(\mathcal{T}_{H \circ \theta(z)}\right)$ would be in $\boldsymbol{\Delta}_{1}^{1}=\boldsymbol{\Sigma}_{1}^{1} \cap \boldsymbol{\Pi}_{1}^{1}$ which is the class of Borel sets by Suslin's Theorem. Thus $\Sigma^{\omega, \omega}-L^{B}\left(\mathcal{T}_{H \circ \theta(z)}\right)$ cannot be Büchi-recognizable because it is not a $\Sigma_{1}^{1}$-subset of $\Sigma^{\omega, \omega}$ and $T S\left(\Sigma^{\omega, \omega}\right) \subseteq \Sigma_{1}^{1} \subseteq \boldsymbol{\Sigma}_{1}^{1}$, see [i]].

Finally, using the reduction $H \circ \theta$, the result follows from the $\Pi_{2}^{1}$-completeness of the universality problem for $\omega$-languages of Turing machines. 
We gave in [9] a solution to a question of [1], showing that all languages of infinite pictures which are accepted row by row by Büchi or Choueka automata reading words of length $\omega^{2}$ are Büchi recognized by a finite tiling system, but the converse is not true. Then we showed that one cannot decide whether a given Büchi-recognizable language of infinite pictures is accepted row by row by a Büchi or Choueka automaton reading words of length $\omega^{2}$. We are going to show now that this decision problem is actually also $\Pi_{2}^{1}$-complete.

Recall that an $\omega^{2}$-word $x$ over the alphabet $\Sigma$ is a sequence of length $\omega^{2}$ of letters in $\Sigma$. It is denoted by $(x(i))_{0 \leq i<\omega^{2}}=x(0) . x(1) . x(2) \ldots x(i) \ldots$, where for all $i, 0 \leq i<\omega^{2}, x(i)$ is a letter in $\Sigma$.

The set of $\omega^{2}$-words over $\Sigma$ is denoted by $\Sigma^{\omega^{2}}$. An $\omega^{2}$-language over $\Sigma$ is a subset of $\Sigma^{\omega^{2}}$.

To define a notion of acceptance row by row of an $\omega$-picture we first associate, to an infinite picture $p \in \Sigma^{\omega, \omega}$, an $\omega^{2}$-word $\bar{p} \in \Sigma^{\omega^{2}}$ which is defined by $\bar{p}(\omega \cdot n+m)=p(m+1, n+1)$ for all integers $n, m \geq 0$.

This can be extended to languages of infinite pictures: for $L \subseteq \Sigma^{\omega, \omega}$ we denote $\bar{L}=\{\bar{p} \mid p \in L\}$ so $\bar{L}$ is an $\omega^{2}$-language over $\Sigma$.

We refer the reader to [9] for a precise definition of generalized Büchi automaton acceptings words of ordinal length. We recall now the following definition.

Definition 5.14. A language of infinite pictures $L \subseteq \Sigma^{\omega, \omega}$ is accepted row by row by an ordinal Büchi automaton if and only if the $\omega^{2}$-language $\bar{L}$ is regular, i.e. is accepted by an ordinal Büchi automaton. We denote $B A\left(\Sigma^{\omega, \omega}\right)$ the set of languages $L \in \Sigma^{\omega, \omega}$ such that $\bar{L}$ is regular.

We can now state the following result.

Theorem 5.15. The problem to determine whether a given Büchi-recognizable language of infinite pictures is accepted row by row by an ordinal Büchi automaton, is $\Pi_{2}^{1}$-complete, i.e. :

$$
\left\{z \in \mathbb{N} \mid \bar{L}^{B}\left(\mathcal{T}_{z}\right) \text { is regular }\right\} \text { is } \Pi_{2}^{1} \text {-complete. }
$$

Proof. Recall that, for each language of infinite pictures which is accepted row by row by a Büchi automaton reading words of length $\omega^{2}$, it was constructed in [9] a Muller tiling system accepting it. Then, using [1], Theorem 1], one can effectively construct a Büchi tiling system accepting the same language. The set $T_{R}$ of indices of Büchi tiling systems constructed from the proof of [9, Theorem 4.1] and [1], Theorem 1] is easily seen to be recursive. Notice that $T_{R}$ does not contain all indices of Büchi tiling systems accepting languages in $B A\left(\Sigma^{\omega, \omega}\right)$. But for each language $L$ in $B A\left(\Sigma^{\omega, \omega}\right)$ there is an index $z \in T_{R}$ such that $L=L^{B}\left(\mathcal{T}_{z}\right)$.

We can then express " $\bar{L}^{B}\left(\mathcal{T}_{z}\right)$ is regular" by the formula " $\exists y\left[\left(y \in T_{R}\right)\right.$ and $L^{B}\left(\mathcal{T}_{z}\right)=L^{B}\left(\mathcal{T}_{y}\right)$ ". This is a $\Pi_{2}^{1}$-formula because " $L^{B}\left(\mathcal{T}_{z}\right)=L^{B}\left(\mathcal{T}_{y}\right)$ " can be expressed by a $\Pi_{2}^{1}$-formula and the quantifier $\exists y$ is of type 0 .

To prove completeness we can use the same reduction $H \circ \theta$ as in the proof of Theorem 5.11. We have seen that there are two cases.

First case. $L\left(\mathcal{M}_{z}\right)=\Sigma^{\omega}$ and then $L^{B}\left(\mathcal{T}_{H \circ \theta(z)}\right)=\Sigma^{\omega, \omega}$. In particular, $L^{B}\left(\mathcal{T}_{H \circ \theta(z)}\right)=\Sigma^{\omega, \omega}$ is accepted 
row by row by an ordinal Büchi automaton.

Second case. $L\left(\mathcal{M}_{z}\right) \neq \Sigma^{\omega}$. Then we have seen that $L^{B}\left(\mathcal{T}_{H \circ \theta(z)}\right)$ is not a Borel set. Thus in that case $\bar{L}^{B}\left(\mathcal{T}_{H \circ \theta(z)}\right)$ is not a regular $\omega^{2}$-language because otherwise $L^{B}\left(\mathcal{T}_{H \circ \theta(z)}\right)$ would be a Borel set (of rank smaller than or equal to 5), see [9, Proposition 4.2].

Finally, using the reduction $H \circ \theta$, the completeness result follows from the $\Pi_{2}^{1}$-completeness of the universality problem for $\omega$-languages of Turing machines.

\section{Concluding Remarks}

We have given in this paper the exact degree of numerous natural decision problems for recognizable languages of infinite pictures. This way we have given examples of natural highly undecidable problems which are complete at the first or at the second level of the analytical hierarchy. Notice that many examples of $\Sigma_{1}^{1}$-complete problems are already known, such as the recurring tiling problem, see for instance [12, 13, 14]. But it seems that very few natural problems, except some problems about $\omega-$ languages of Turing machines, are known to be $\Pi_{2}^{1}$-complete. One of the motivation of Castro and Cucker in [3] was actually to "give natural complete problems for the lowest levels of the analytical hierarchy which constitute an analog of the classical complete problems given in recursion theory for the arithmetical hierarchy". So we have added in this paper many new examples which complete the work of [3].

Notice that in another paper we have also given many natural $\Pi_{2}^{1}$-complete problems about the infinite behaviour of very simple finite machines like 1-counter automata or 2-tape automata, [10].

We hope also that our results could be useful in other connected areas, for instance in the study of the infinite behaviour of cellular automata, [25, 6].

\section{Acknowledgements.}

Thanks to the anonymous referees for useful comments on a preliminary version of this paper.

\section{References}

[1] Altenbernd, J.-H., Thomas, W., Wöhrle, S.: Tiling Systems over Infinite Pictures and Their Acceptance Conditions, Proceedings of the 6th International Conference Developments in Language Theory, DLT 2002, 2450, Springer, 2003.

[2] Ballier, A., Jeandel, E.: Tilings and Model Theory, Proceedings of the Journées Automates Cellulaires 2008, Uzès, France, 2008.

[3] Castro, J., Cucker, F.: Nondeterministic $\omega$-Computations and the Analytical Hierarchy, Journal Math. Logik und Grundlagen d. Math, 35, 1989, 333-342.

[4] Cohen, R., Gold, A.: $\omega$-computations on Turing machines, Theoretical Computer Science, 6, 1978, 1-23.

[5] Darondeau, P., Yoccoz, S.: Proof Systems for Infinite Behaviours, Information and Computation, 99(2), 1992, 178-191. 
[6] Delorme, M., Mazoyer, J., Eds.: Cellular Automata, a parallel Model, Kluwer Academic, 1994.

[7] Engelfriet, J., Hoogeboom, H. J.: X-automata on $\omega$-words, Theoretical Computer Science, 110(1), 1993, $1-51$.

[8] Finkel, O.: Borel hierarchy and omega context free languages, Theoretical Computer Science, 290(3), 2003, 1385-1405.

[9] Finkel, O.: On recognizable languages of infinite pictures, International Journal of Foundations of Computer Science, 15(6), 2004, 823-840.

[10] Finkel, O.: Highly Undecidable Problems for Infinite Computations, 2008, Preprint, 32 pages, submitted.

[11] Giammarresi, D., Restivo, A.: Two-Dimensional Languages, in: Handbook of formal languages, Vol. 3, Springer, Berlin, 1997, 215-267.

[12] Harel, D.: Recurring Dominoes: Making the Highly Undecidable Highly Understandable, Fundamentals of Computation Theory, Proceedings of the 1983 International FCT-Conference, Borgholm, Sweden, August 21-27, 1983, 158, Springer, 1983.

[13] Harel, D.: Effective transformations on infinite trees, with applications to high undecidability, dominoes, and fairness, Journal of the ACM, 33(1), 1986, 224-248.

[14] Hirst, T., Harel, D.: Taking It to the Limit: On Infinite Variants of NP-Complete Problems, Journal of Computer and System Sciences, 53(2), 1996, 180-193.

[15] Kechris, A. S.: Classical descriptive set theory, Springer-Verlag, New York, 1995, ISBN 0-387-94374-9.

[16] Landweber, L.: Decision problems for $\omega$-automata, Mathematical Systems Theory, 3(4), 1969, 376-384.

[17] Lescow, H., Thomas, W.: Logical specifications of infinite computations, A Decade of Concurrency (J. W. de Bakker, W. P. de Roever, G. Rozenberg, Eds.), 803, Springer, 1994.

[18] Moschovakis, Y. N.: Descriptive set theory, North-Holland Publishing Co., Amsterdam, 1980, ISBN 0-44485305-7.

[19] Perrin, D., Pin, J.-E.: Infinite words, automata, semigroups, logic and games, vol. 141 of Pure and Applied Mathematics, Elsevier, 2004.

[20] Prasad Sistla, A.: On Verifying That A Concurrent Program Satisfies A Nondeterministic Specification, Information Processing Letters, 32(1), 1989, 17-23.

[21] Rogers, H.: Theory of Recursive Functions and Effective Computability, McGraw-Hill, New York, 1967.

[22] Staiger, L.: Hierarchies of recursive $\omega$-languages, Elektronische Informationsverarbeitung und Kybernetik, 22(5-6), 1986, 219-241, ISSN 0013-5712.

[23] Staiger, L.: w-languages, in: Handbook of formal languages, Vol. 3, Springer, Berlin, 1997, 339-387.

[24] Thomas, W.: Automata on infinite objects, in: Handbook of Theoretical Computer Science (J. van Leeuwen, Ed.), vol. B, Formal models and semantics, Elsevier, 1990, 135-191.

[25] Wolfram, S., Ed.: Theory and Applications of Cellular Automata, World Scientific Press, 1986. 